\title{
Análisis de la expresión y función de los sustratos del receptor de insulina
}

\author{
Paola Valentina Valero Ávila \\ pvalero@uji.es \\ Ana María Sánchez Pérez \\ sanchean@med.uji.es
}

\section{Resumen}

La diabetes y la resistencia a insulina están asociada a un mayor riesgo de aparición de enfermedades neurodegenerativas. La señalización de insulina está mediada por los sustratos del receptor de insulina (IRS). Alteraciones en la expresión de estas proteínas pueden resultar en resistencia a la insulina. Estudios previos del laboratorio muestran cambios en los niveles del mRNA del IRS1 y del IRS2 en situación de síndrome metabólico.

Este trabajo pretende caracterizar la región promotora que regula la expresión de estos genes. Para ello, hemos analizado, mediante herramientas bioinformáticas, las secuencias reguladoras, es decir, los sitios consenso de unión a factores de transcripción, presentes en los promotores de estos genes de rata. Por otro lado, para observar la funcionalidad del promotor in vitro, se pretende clonar la región promotora y estudiar su actividad con la expresión de un gen reportero.

El análisis bioinformático muestra prevalentemente la unión de la familia de factores HES al promotor de IRS2 y no en IRS1. Además, la secuencia promotora clonada muestra funcionalidad cuando se transfectan células heterólogas in vitro.

Las diferencias en el promotor pueden explicar el diferente comportamiento de estos mRNA y es la base para estudios futuros de este factor de transcripción en situaciones de resistencia a insulina. Se podría mejorar la funcionalidad del promotor empleando elementos potenciadores de la transcripción como enhancers, para en un futuro poder estudiar su actividad in vivo y bajo diferentes condiciones.

Palabras clave: resistencia a insulina, promotor IRS1/2, bioinformática, clonación, cultivos celulares.

\section{Abstract}

Diabetes and resistance to the disease are associated with a risk of neurodegenerative diseases. Insulin signaling is mediated by insulin receptor 
substrates (IRS). Alterations in the expression of these proteins can result in an insulin resistance. Previous studies of the laboratory showed changes in the levels of mRNA of IRS1 and IRS2 in situation of metabolic syndrome. In this work we aim to study the promoter activity that regulates the expression of these genes, as well as the function of these proteins in vitro.

Bioinformatic tools have been used to identify the regulatory sequences present in the promoters. On the other hand, to determine the functionality of the promoter in vitro, it was necessary to clone it into a plasmid, which took into account the genomic DNA, the amplification of the fragment by PCR, the ligation in the final plasmid and the verification in cells in vitro

Bioinformatic analysis shows the binding of the family of HES factors to the IRS2 promoter and not to IRS1. In addition, the promoter sequence shows a small amount of in vitro activity, due to the low efficiency in transfection.

The differences in the promoter may explain the different behavior of these mRNAs, and this is the basis for future studies of this transcription factor in situations of insulin resistance. The functionality of the promoter could be improved by using transcription enhancing elements as enhancers, to be able to study it in vivo and under different conditions.

Keywords: Insulin resistance, IRS1/2 promoter, Bioinformatics, Cloning, Cell cultures.

\section{Introducción}

La insulina media su acción a través de la unión a su receptor, que tiene actividad tirosin kinasa. Esto quiere decir que, cuando la insulina se une a su receptor, este se activa y se autofosforila en residuos de tirosina y además fosforila a sus sustratos. Entre las proteínas diana del receptor de insulina destacan los demonimados sustratos del receptor de insulina (IRS) cuyas formas prioritarias en cerebro son IRS1 e IRS2. En condiciones fisiológicas normales, los IRS se fosforilan en residuos aminoacídicos de tirosina; no obstante, en situaciones de neuroinflamación, como por ejemplo la enfermedad de Alzheimer, hay una señalización de insulina diferente a la habitual, que concluye con la fosforilación de los IRS en residuos de serina, lo cual favorece la muerte neuronal (Bedse, Di Domenico, Serviddio y Cassano 2015). En algunos casos se han descrito alteraciones de los niveles de IRS1 e IRS2 en modelos animales de Alzheimer (Chen et al. 2013; Zhang et al. 2015) o cerebros posmortem de pacientes (Moloney et al. 2010).

Estudios previos han demostrado que la neuroinflamación producida por una dieta rica en contenido graso produce la disminución de la expresión del mRNA y proteína IRS2 y un leve incremento del IRS1 en cerebro, sin afectar los niveles de expresión del receptor de insulina (Ribes-Navarro et al. 2019). Además, la actividad promotora es una forma de regular los niveles de mRNA. El promotor se puede definir como la secuencia inmediatamente anterior al inicio de la transcripción (figura 1). 


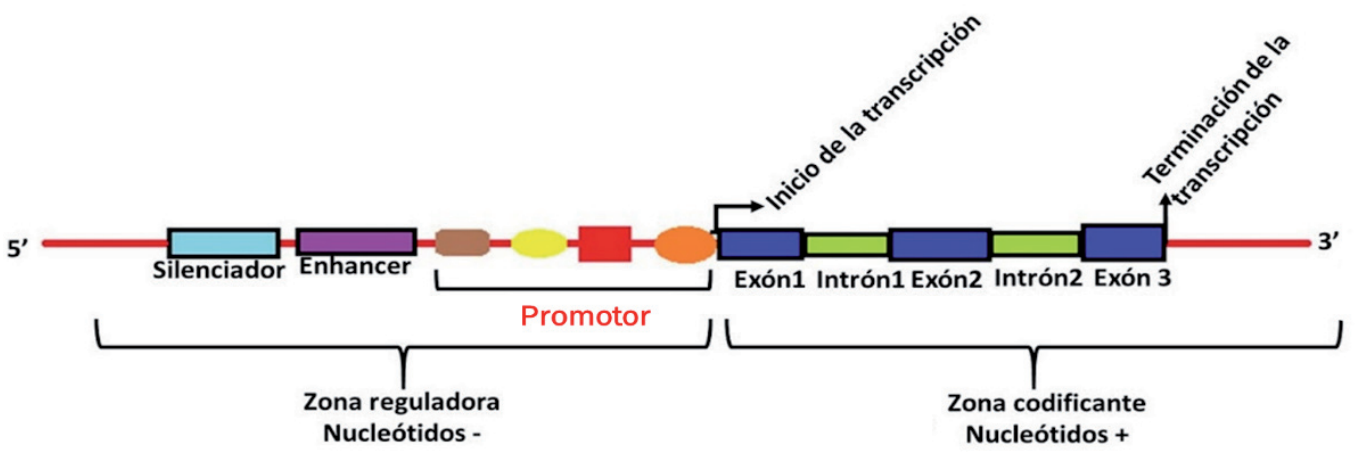

Figura 1. Esquema general de un gen. A partir del inicio de la transcripción (TS), los nucleótidos se cuentan $+y$ en dirección 5 ' en la región reguladora lo nucelotidos son - .

El objetivo del presente estudio es caracterizar la secuencia promotora de los genes IRS1 e IRS2 en humano y rata. Además, clonar el promotor de IRS1 de rata, dirigiendo la expresión de un gen reportero fluorescente como medida de su actividad.

Gracias a este estudio, se podrá determinar bajo qué condiciones se activa el promotor y también de qué modo las situaciones de inflamación pueden alterar la expresión génica. Además, este tipo de herramienta nos permitirá analizar in vivo en qué tipos celulares y áreas cerebrales se expresan estos genes y estudiar variaciones en su expresión en diferentes condiciones de neuroinflamación e incluso durante el desarrollo y el envejecimiento. Con ello, se podrá conocer la regulación del gen en la forma fisiológica y en condiciones patológicas y permitirá desarrollar estrategias dirigidas a revertir aquellas situaciones anómalas.

\section{Métodos}

\section{Análisis bioinformático de la secuencia promotora}

Las potenciales secuencias promotoras de los genes IRS1 de humano y rata fueron obtenidas en la base de datos Ensembl, con los siguientes códigos ENSG00000169047 y ENSRNOG00000014597 y las secuencias de IRS2 humano y rata con los códigos ENSG00000185950 y ENSRNOG00000023509, respectivamente.

Mediante el programa Blast se realizó un alineamiento de las secuencias promotoras de las dos especies para determinar su grado de homología y, a continuación, se analizaron las secuencias consenso, empleando el sotfware gratuito Gene Promoter Miner (figura 5).

Además, se efectuó un análisis computacional para determinar secuencias consenso de los sitios de unión a factores de transcripción (TF) en los promotores IRS1/2 humano y de rata, mediante el programa Genomatix MatInspector (figura 6).

\section{Clonación del promotor IRS1de rata}

Se extrajo el ADN genómico de un cultivo confluyente de células B50 (ECACC 85042302), mantenidas en medio DMEM rico en glucosa, empleando el kit Purelink ${ }^{T M}$ Genomic DNA Mini kit (invitrogen) de acuerdo con las instrucciones del fabricante. 
Para la amplificación del promotor se diseñaron primers que abarcaran un fragmento de $1 \mathrm{~Kb}$ justo al inicio del inicio de la transcripción. Las secuencias diseñadas fueron:

\section{Forward:5'TAAGCAGAATTCTCTCCCTACCAGGGCCTC3'}

\section{Reverse:5'TGCTTAGGGCCCCTCAATCGCAGAGACCGC3'}

Ambos primers poseen en su extremo 5' sitios de corte para las enzimas de restricción; EcoRI en el forward y Apal en el reverse.

Tras la reacción de amplificación en cadena (PCR), se realizó una electroforesis en gel de Agarosa al $1 \%$ y tampón TAE 1X. Se purificó la banda específica empleando el kit Extractme DNA gel-out kit (blirt) según las especificaciones del fabricante.

Se procedió a la digestión enzimática del plásmido vector pJET 1.2/blunt (CloneJET PCR Cloning Kit; Thermo Fisher Scientific) (figura 2) y del inserto (el promotor IRS1 de rata producto de la PCR), empleando las enzimas EcoRI y APAI, manteniendo la reacción durante $1 \mathrm{~h} 30$ minutos a $37^{\circ} \mathrm{C}$, y luego se purificaron las correspondientes bandas a partir del gel de agarosa.

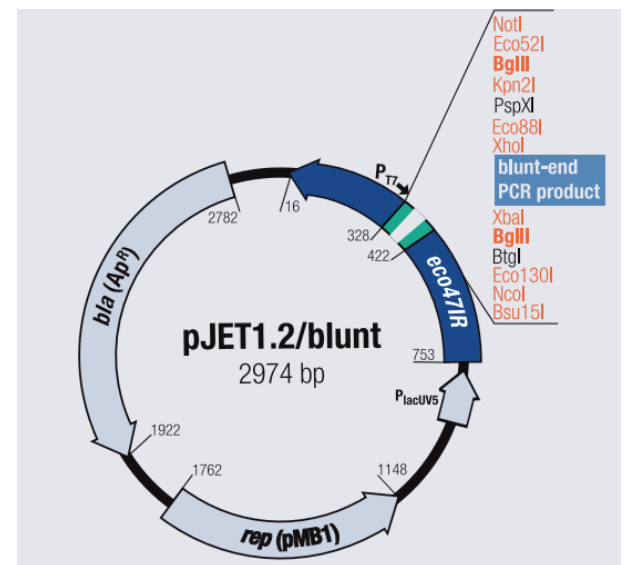

Figura 2. Plásmido comercial pJET 1.2/blunt (Thermo Fisher Scientific) junto con los sitios de restricción presentes en su multicloning site.

La ligación entre inserto y el plásmido vector se realizó empleando la ligasa T4 y, posteriormente, se transformó en la línea celular bacteriana Endura ${ }^{\mathrm{TM}}$. Las bacterias se sembraron en placas de agar con ampicilina al $1 \%$, para seleccionar las colonias que habían introducido el vector. Tras 24 horas, se seleccionaron un par de colonias positivas, que crecieron en cultivo líquido durante 16 horas a $37^{\circ} \mathrm{C}$ y en agitación, y se procedió a la extracción del plásmido mediante el kit comercial NZYMiniprep (nzytech). Una vez el promotor estaba en el plásmido pJET, el siguiente paso fue cortar y extraer el promotor mediante la digestión con los mismos enzimas que liberan el inserto. El plásmido de destino pAC-GFP1-1 (Clontech Laboratories, Inc.) (figura 3) se cortó con los mismos enzimas, para generar los extremos cohesivos. Este vector contiene el gen reporter EGFP, que se expresará bajo la actividad del promotor que se introduzca delante. En este caso el promotor IRS1 de rata. 


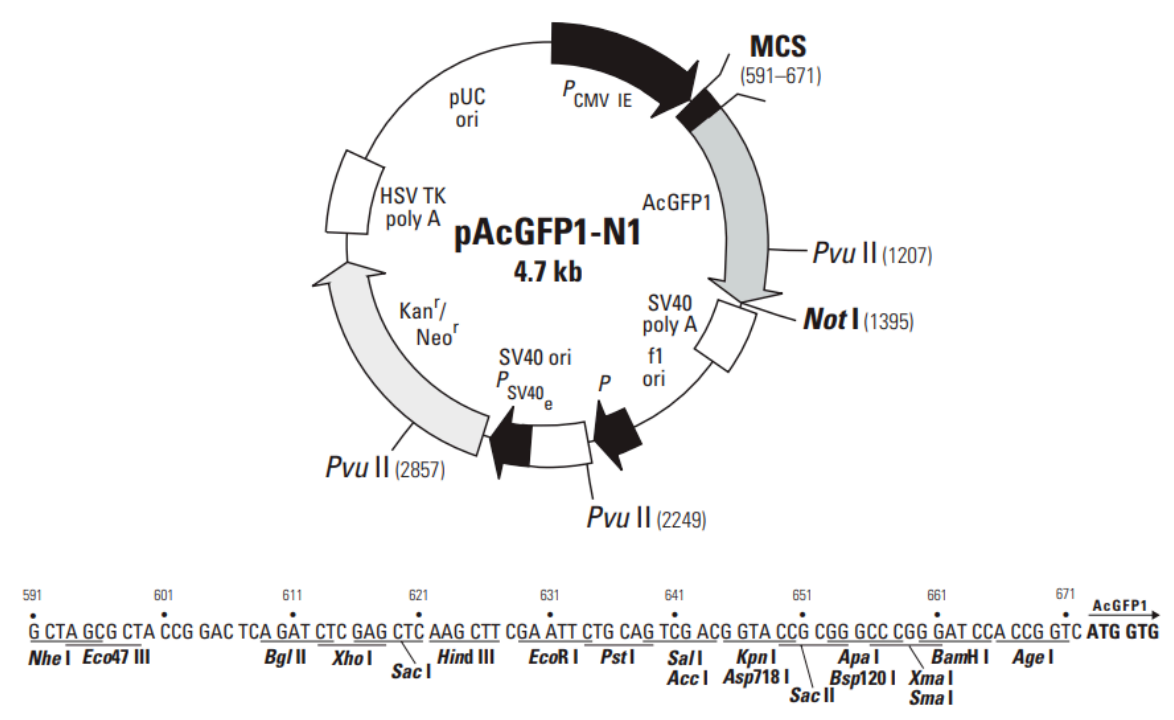

Figura 3. Plásmido comercial pAC-GFP1-1 (Clontech Laboratories, Inc.), junto con los sitios de restricción presentes en su multicloning site.

Una vez purificiado el vector abierto y el inserto, ambos se ligaron y el constructo resultante se volvió a introducir en bacterias. En este caso se añadió un control negativo de la transformación, el constructo solo, para controlar posibles colonias de vector recircularizado. Al igual que anteriormente, se procedió a la selección de las colonias positivas por selección con antibiótico. Se prosiguió con el crecimiento de la colonia y miniprep del constructo final, el pAC-promolRS1-GFP1-1.

Prueba de la funcionalidad del promotor in vitro

Se cultivaron células HEK293 en medio DMEM hasta una confluencia del 70-80 \%, luego se llevó a cabo por separado la transfección del pAC-promoIRS1-GFP1-1 y del plásmido control pAAV-GFP (figura 4) mediante la técnica de cloruro de calcio. A las $48 \mathrm{~h}$ se procedió a fotografiar la fluorescencia celular.

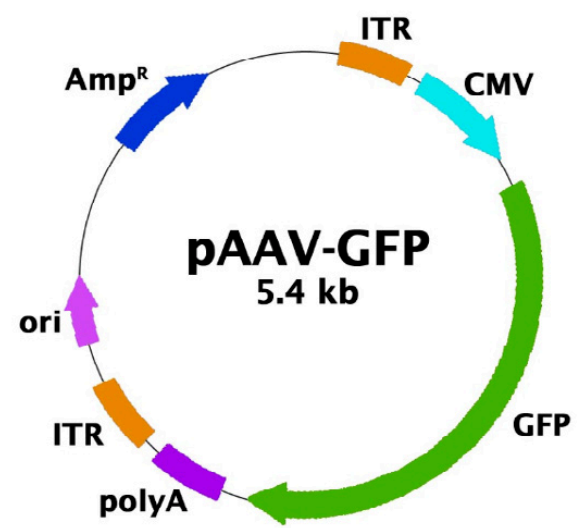

Figura 4. Plásmido comercial pAAV-GFP (Cell Biolabs, Inc.). 


\section{Resultados}

El análisis bioinformático de las secuencias consenso típicas en los promotores (figura 5) muestra que todos los promotores contienen múltiples GC boxes. Sin embargo, en el caso de humanos se encuentran más próximas al sitio de inicio de la transcripción (0) que en el caso de rata, cuyas posiciones aparecen más distribuidas a lo largo de la secuencia promotora. Además, el promotor de IRS1 de rata es el único que no contiene CCAAT box.

Por otro lado, hay una alta homología, en torno al 79-80\%, en las parejas de promotores (figura 5), en una parte de la secuencia (48\% para el promotor de IRS1 y $34 \%$ para el promotor de IRS2).

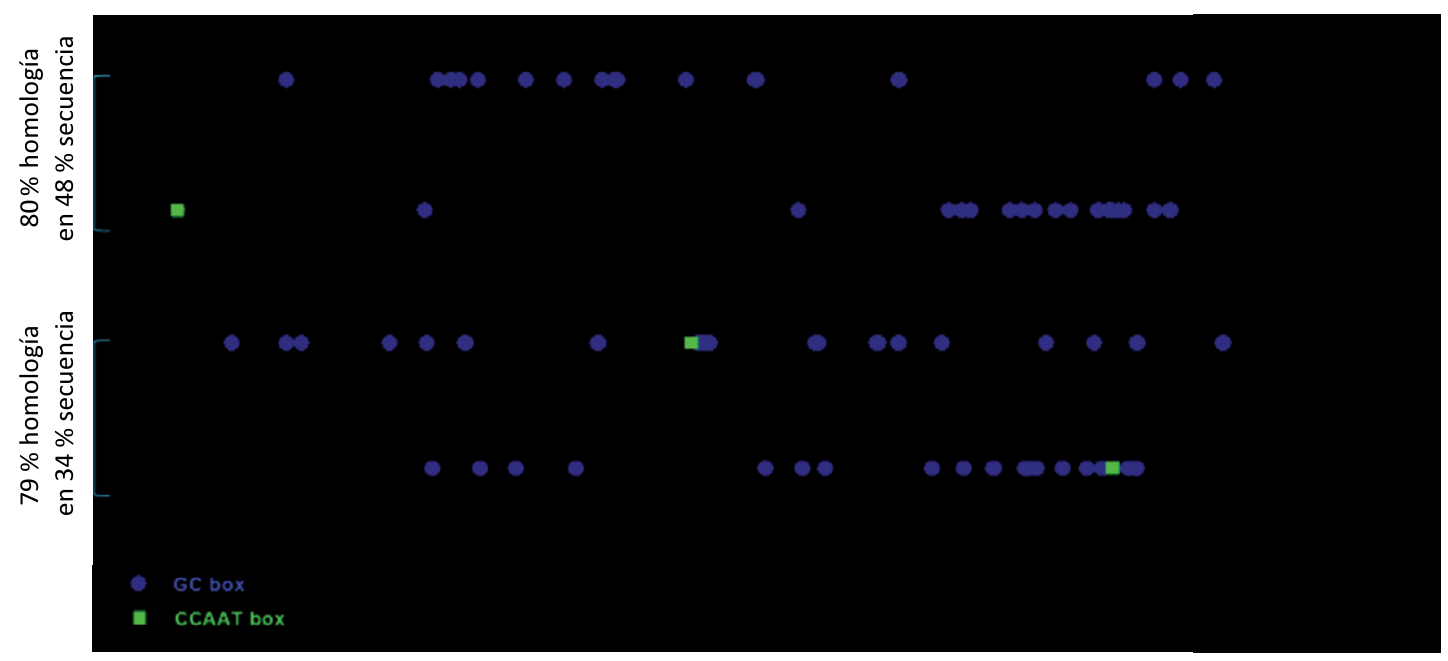

Figura 5. Análisis de las secuencias consenso en los promotores IRS1/2 de rata y humano, así como la homología de secuencia entre los promotores IRS1 e IRS2 de las dos especies.

De entre todos los posibles factores de transcripción que pueden unirse a la secuencia promotora, se seleccionaron aquellos que presentaban cinco o más sitios de unión en el promotor y que, además, se unían con una alta probabilidad. Se adoptaron estos criterios siguiendo la hipótesis de que, si un factor de transcripción tiene más sitios de unión en el promotor, tendrá por tanto un peso mayor en su regulación.

Se determinó que los factores de transcripción comunes a los promotores de IRS1 e IRS2 eran E2F y PLAG. En cambio, el promotor de IRS2, tanto en rata como humano, presenta un mayor número de sitios de unión para HES1 en comparación con los promotores de IRS1 en sus respectivas especies (figura 6). 


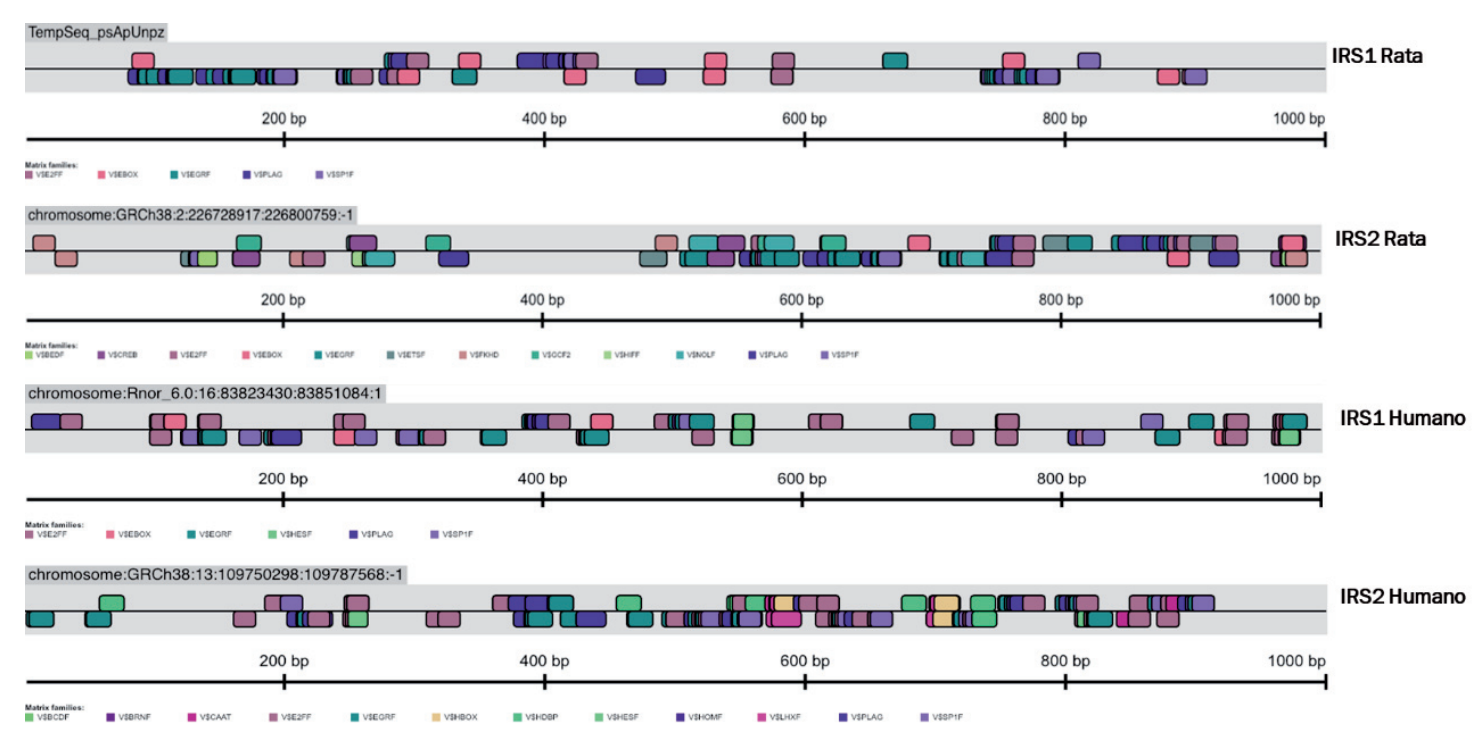

Figura 6. Análisis de los sitios de unión para factores de transcripción en los promotores IRS1/2 de rata y humano.

La transfección heteróloga del pAC-promoIRS1-GFP1-1 en células humanas HEK293 muestra funcionalidad de la secuencia promotora, puesto que hay expresión del gen reportero GFP. Sin embargo, su actividad es baja en este tipo celular en comparación con el control (figura 7).

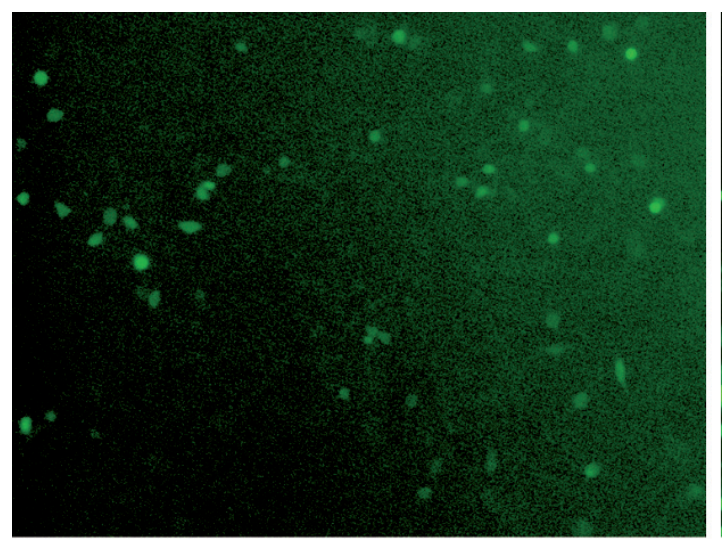

Hek293T 72h tras la transfección con pACpromolRS1-GFP1-1.

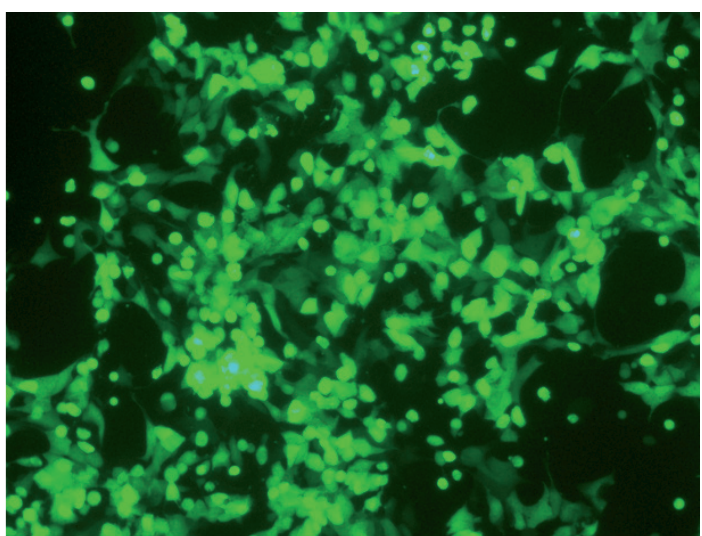

Hek293T 72h tras la transfección con pAAVGFP control.

Figura 7. Comparación entre la transfección con cloruro de calcio del pAC-promolRS1GFP1-1 frente al pAAV-GFP control en células HEK293T a las 72 horas.

\section{Discusión y conclusiones}

Gracias al presente estudio podría analizarse desde otra perspectiva la regulación de la vía de señalización de insulina, la cual, como se ha indicado anteriormente, parece estar afectada en enfermedades de carácter neurodegenerativo. Por ende, la 
regulación del promotor de los IRS es muy interesante, ya que su modulación podría ser un potencial tratamiento frente a la resistencia a insulina o enfermedades neurodegenerativas.

En conjunto, el análisis informático indica que las secuencias obtenidas, con alta probabilidad, son secuencias promotoras, porque hay un gran número de elementos consenso típicos (Espinar, Schikora Tamarit, Domingo y Carey 2018; HernandezGarcia y Finer 2014); además, la secuencia promotora de IRS1 de rata es funcional in vitro. Por otra parte, la alta homología entre las secuencias en las distintas especies indica que estas secuencias tienen una importancia biológica, ya que se han conservado a lo largo de la evolución (Christin, Weinreich y Besnard 2010). Por otro lado, el factor HES1 se ha descrito que se produce en el cerebro adulto de ratón tras la estimulación neuronal y su inactivación en neuronas excitatorias produce ansiedad y miedo de forma concominante con una alta excitabilidad neuronal en la amigdala, mientras que la inactivación de Hes1 en nueronas inhibitorias produce un incremento de la sociabilidad y tendencias preservativas (Matsuzaki, Yoshihara, Ohtsuka y Kageyama 2019). Nosotros, por nuestra parte, hemos observado que Hes 1 parece tener un papel distintivo en la regulación del promotor de IRS2, con lo cual podríamos estudiar bajo qué condiciones se une este factor de transcripción y en qué situaciones se altera su unión, incluso posibles metilaciones, para analizar la diferente regulación de IRS2 e IRS1 y, posteriormente, poder modular in vivo la regulación del promotor, observando los efectos finales en conducta. Otro dato interesante es la distinta distribución de las islas CpG entre humanos y ratas, que son lugares de unión a factores como el Sp1, además de sitios potenciales de metilación (Zhu et al. 2003), aunque estas islas también parecen regular la actividad promotora directamente (Hartl et al. 2019), con lo cual esto abre otro campo de estudio acerca de la regulación de los promotores de los sustratos del receptor de insulina.

Hasta la fecha, la principal limitación por la cual no se ha podido realizar la transfección exitosa del promotor en lineas celulares de rata ha sido la baja eficiencia de transfección por parte de esta línea celular, con lo cual ha sido necesaria la expresión heteróloga del promotor de IRS1 de rata en células HEK293 (humana), la cual tiene una alta eficiencia de transfección (Thomas y Smart 2005). Los resultados sugieren que el promotor es muy poco activo en esta linea celular dada la baja intensidad de la señal fluorescente. Además, la baja actividad promotora de este constructo en las células HEK293 podría ser consistente con la diferencia de homología $(20 \%)$ que muestra el análisis bioinformático de las secuencias consenso de los promotores de rata y humano, a pesar de la alta similitud de los sitios de unión para factores de transcripción en ambas especies.

En conclusión, consideramos que esta vía de estudio de la actividad promotora será una herramienta muy útil para, en un futuro, poder establecer las diferencias de expresión de estos genes en distintas situaciones fisiopatológicas, además de permitir probar cómo la expresión de IRS1 e IRS2 se afecta de forma diferencial en distintas situaciones y tipos celulares, en el desarrollo o en el envejecimiento. Incluso se podría modular la regulación del promotor in vivo, introduciendo el promotor mutado en estos sitios de regulación directamente en el cerebro de animales sanos (o modelos de neurodegeneración), a través de la técnica CRISPR-Cas9, y observar el efecto cognitivo que causaría. 


\section{Referencias bibliográficas}

Bedse, Gaurav, Fabio Di Domenico, Gaetano Serviddio y Tommaso Cassano. 2015. «Aberrant insulin signaling in Alzheimer's disease: Current knowledge». Frontiers in Neuroscience, 9: 204.

Chen, Yanxing, Zhihou Liang, Julie Blanchard, Chun-Ling Dai, Shenggang Sun, Moon H. Lee, Inge Grundke-Iqbal, Khalid Iqbal, Fei Liu y Cheng-Xin Gong. 2013. «A non-transgenic mouse model (icv-STZ mouse) of Alzheimer's disease: similarities to and differences from the transgenic model (3xTg-AD mouse)». Molecular Neurobiology, 47(2): 711-725.

Christin, Pascal-Antoine, Daniel M. Weinreich y Guillaume Besnard. 2010. «Causes and evolutionary significance of genetic convergence». Trends in Genetics, 26(9): 400-405.

Espinar, Lorena, Miquel Àngel Schikora Tamarit, Júlia Domingo y Lucas B. Carey. 2018. "Promoter architecture determines cotranslational regulation of mRNA». Genome Research, 28(4): 509-518.

Hartl, Dominik, Arnaud R. Krebs, Ralph S. Grand, Tuncay Baubec, Luke Isbel, Christiane Wirbelauer, Lukas Burger y Dirk Schübeler. 2019. «CG dinucleotides enhance promoter activity independent of DNA methylation». Genome Research, 29(4): 554-563.

Hernandez-Garcia, Carlos M. y John J. Finer. 2014. «Identification and validation of promoters and cis-acting regulatory elements». Plant Science, 217-218: 109119.

Matsuzaki, Tadanobu, Toru Yoshihara, Toshiyuki Ohtsuka y Ryoichiro Kageyama. 2019. "Hes1 expression in mature neurons in the adult mouse brain is required for normal behaviors». Scientific Reports, 9(1): 8251.

Moloney, Aileen M., Rebecca J. Griffin, Suzanne Timmons, Rosemary O'Connor, Rivka Ravid y Cora O'Neill. 2010. «Defects in IGF-1 receptor, insulin receptor and IRS-1/2 in Alzheimer's disease indicate possible resistance to IGF-1 and insulin signalling». Neurobiology of Aging, 31(2): 224-243.

Ribes-Navarro, Alberto, Mariam Atef Othman, Sandra Sánchez-Sarasúa, María Teresa Beltrán Bretones, Francisco E. Olucha-Bordonau y Ana María Sánchez-Pérez. 2019. "Abscisic Acid Supplementation Rescues High Fat Diet-Induced Alterations in Hippocampal Inflammation and IRSs Expression». Molecular Neurobiology, 56(1): 454-464.

Thomas, Philip y Trevor G. Smart. 2005. «HEK293 cell line: A vehicle for the expression of recombinant proteins». Journal of Pharmacological and Toxicological Methods, 51(3): 187-200.

Zhang, Yonglan, Fei Yin, Jianhui Liu, Zixuan Liu, Lixia Guo, Zhining Xia y Jeffrey Zidichouski. 2015. "Geniposide attenuates insulin-deficiency-induced acceleration of $\beta$-amyloidosis in an APP/PS1 transgenic model of Alzheimer's disease». Neurochemistry International, 89: 7-16.

Zhu, Wei-Guo, Kanur Srinivasan, Zunyan Dai, Wenrui Duan, Lawrence J. Druhan, Haiming Ding, Lisa Yee, Miguel A. Villalona-Calero, Christoph Plass y Gregory A. Otterson. 2003. «Methylation of Adjacent CpG Sites Affects Sp1/Sp3 Binding and Activity in the p21Cip1 Promoter». Molecular and Cellular Biology, 23(12): 4056-4065. 